\title{
Differences in DNA methylation of white blood cell types at birth and in adulthood reflect postnatal immune maturation and influence accuracy of cell type prediction
}

Meaghan J Jones ${ }^{1,2}$, Louie Dinh ${ }^{1,2}$, Hamid Reza Razzaghian ${ }^{2,3}$, Olivia de Goede ${ }^{4}$, Julia L Maclsaac ${ }^{1,2}$, Alexander M. Morin ${ }^{1,2}$, Kristina Gervin ${ }^{5}$, Raymond Ng ${ }^{6}$, Liesbeth Duijts ${ }^{7}$, Menno C van Zelm ${ }^{8,9}$, Henriëtte A Moll ${ }^{10}$, Robert Lyle ${ }^{5}$, Wendy P Robinson ${ }^{1,2}$, Devin C Koestler $^{11}$, Janine F Felix ${ }^{12}$, Pascal M Lavoie ${ }^{2,3}$, Sara Mostafavi ${ }^{1,2,13}$, Michael S Kobor $^{1,2,13}$

Affiliations:

1. Department of Medical Genetics, University of British Columbia, Vancouver, Canada

2. BC Children's Hospital Research Institute, Vancouver, Canada

3. Department of Pediatrics, University of British Columbia, Vancouver, Canada

4. Department of Genetics, Stanford University, Stanford, CA, USA

5. Department of Medical Genetics, Oslo University Hospital, Oslo, Norway

6. Department of Computer Science, University of British Columbia, Vancouver, Canada

7. Department of Pediatrics, Divisions of Respiratory Diseases and Allergology, and Neonatology, Erasmus MC, University Medical Center Rotterdam, the Netherlands

8. Department of Immunology and Pathology, Central Clinical School, Monash University and The Alfred Hospital, Melbourne, Australia

9. Department of Immunology, Erasmus MC, University Medical Center Rotterdam, Rotterdam, the Netherlands

10. Department of Pediatrics, Erasmus MC-Sophia's Children's Hospital, University Medical Center Rotterdam, Rotterdam, the Netherlands

11. Department of Biostatistics, University of Kansas Medical Center, Kansas City, KS, USA

12. Generation R Study Group, Department of Pediatrics, and Department of Epidemiology Erasmus MC, University Medical Center Rotterdam, Rotterdam, the Netherlands

13. Canadian Institute for Advanced Research, Toronto ON, Canada

\section{Abstract}

Background: DNA methylation profiling of peripheral blood leukocytes has many research applications, and characterizing the changes in DNA methylation of specific white blood cell types between newborn and adult could add insight into the maturation of the immune system. As a consequence of developmental changes, DNA methylation profiles derived from adult white blood cells are poor references for prediction of cord blood cell types from DNA methylation data. We thus examined cell-type specific differences in DNA methylation in leukocyte subsets between cord and adult blood, and assessed the impact of these differences on prediction of cell types in cord blood. 
Results: Though all cell types showed differences between cord and adult blood, some specific patterns stood out that reflected how the immune system changes after birth. In cord blood, lymphoid cells showed less variability than in adult, potentially demonstrating their naïve status. In fact, cord CD4 and CD8 T cells were so similar that genetic effects on DNA methylation were greater than cell type effects in our analysis, and CD8 T cell frequencies remained difficult to predict, even after optimizing the library used for cord blood composition estimation. Myeloid cells showed fewer changes between cord and adult and also less variability, with monocytes showing the fewest sites of DNA methylation change between cord and adult. Finally, including nucleated red blood cells in the reference library was necessary for accurate cell type predictions 55 in cord blood.

Conclusion: Changes in DNA methylation with age were highly cell type specific, and those differences paralleled what is known about the maturation of the postnatal immune system.

Keywords: DNA methylation, immune system, development, cord blood, white blood cells, 450k

\section{Background}

64 One of the main established roles for DNA methylation (DNAm) is in development, 65 where it contributes to the functional maturation, lineage commitment and fate of cells. ${ }^{1}$

66 This has two important implications; the first is that DNAm within a given cell type will 67 change over time as cells differentiate and function develops. ${ }^{2}$ The second is that 68 different types of terminally differentiated cells will have very distinct DNAm profiles. ${ }^{3,4}$

69 As the age of individuals and cell type are two of the major determinants of DNAm variability, analyses of DNAm data must carefully consider those variables ${ }^{5,6}$ Due to an

71 important role of DNAm in development, close assessment of developmental

72 processes, by identifying specific genes or genomic regions that change with age.

73 This is of particular interest in blood, where development of the immune system in early

74 life is linked to long term health outcomes, and so the analysis of the changes in DNAm

75 from birth to adulthood may provide insights into how the immune system matures.

76 Umbilical cord blood is an important and much utilized research tissue, as it is easy to

77 collect from the umbilical cord post-delivery, and thus many studies have assessed

78 DNAm in relatively large numbers of cord blood samples. ${ }^{7,8}$ Cord blood is very distinct

79 from adult blood, as it contains a much greater abundance of nucleated red blood cells

80 (nRBC) expressing unique proteins such as fetal hemoglobin, as well as functionally 
81 distinct myeloid and lymphoid cells ${ }^{9,10}$ These distinct functions reflect the greater

82 reliance on innate immunity in newborns, as adaptive immune cells requires exposure

83 to pathogens in order to mature and generate functional memory ${ }^{11,12}$ Thus, one might

84 expect that innate immune cells such as granulocytes, monocytes, and NK cells, would

85 be more similar over development than adaptive immune cells like B and T cells.

86 However, this relationship is more complex, with differences observed even in the

87 function of innate immunity between newborns and adults, indicating that the

88 functionality of specific innate cell types also changes over development ${ }^{13,12,14}$.

89 These biologically meaningful differences in function are likely to be reflected in DNAm 90 changes over developmental time, and thus can cause complications for the analysis of

91 DNAm data, as computational tools designed for use in adult blood may not function as

92 well for blood from children or newborns. An example of this is cell-type deconvolution,

93 which is one of the major tools used to account for inter-individual differences in cell

94 type composition in mixed tissue samples, such as blood, when more direct measures

95 are not available. ${ }^{15-19}$ Failing to account for these inter-individual differences in cell type

96 composition can lead to both false positive and false negative results in epigenetic

97 association studies, and therefore accurate implementation of this tool in a

98 developmental context is essential. ${ }^{2,5}$ Perhaps not surprisingly, as the most commonly

99 used tool was designed for adult references, it performs poorly on cord blood data. ${ }^{20-24}$

100 In an attempt to address this problem, three different reference datasets for cord blood

101 to create developmental stage specific libraries have been published, but validation

102 studies using these updated references only partially close the gap between adult and

103 cord blood prediction accuracy. ${ }^{20,22,25}$

104 In this study, we compared DNAm profiles of purified leukocyte subsets from cord and

105 adult blood, with the goal of further understanding the biological differences in each cell

106 type as they mature. Using these insights, we then tested specific assumptions of

107 existing deconvolution methods for estimating cell type proportions in cord blood,

108 modified the algorithm to account for the differences between cord and adult, and

109 evaluated the prediction accuracy on two data sets. We showed that differences

110 between cord and adult blood cell types reflected the functional maturation of the 
111 immune system, and these differences must be incorporated into the design of methods

112 to be used on DNAm data.

\section{Results}

115 Cell type-specific DNA methylation in adult and cord blood

116 Previous reports have shown that adult references poorly predict cell types in cord

117 blood. 20-22 We hypothesized that differences in DNAm between cord and adult blood

118 might impact the performance of cell type deconvolution, and so compared patterns of

119 DNAm in the adult and cord blood reference data sets. ${ }^{3,20,25}$ In order to take advantage

120 of as many samples as possible, we combined two previously published cord blood data

121 sets, and compared them to a publicly available adult blood data set (Table 1). ${ }^{3,20,25}$ All

122 three data sets were generated from Fluorescence Activated Cell Sorting (FACS)-

123 isolated white blood cell types from healthy donors, resulting in 6 sets of adult blood and

12418 sets of matched 450k cord blood DNAm profiles. All three sets were combined and

125 processed together, and after processing and filtering, 428,688 probes remained.

126 Visualization by hierarchical clustering of all CpGs analyzed showed that samples

127 grouped first by myeloid (granulocytes, monocytes) versus lymphoid (B, $T$, NK cells)

128 lineage, then by age, and finally by specific cell type (Figure 1A). Adult lymphocytes

129 were the most distinct group, followed by nRBCs. In cord blood samples, CD4 and CD8

130 T cells clustered in one large group, paired by individual as opposed to cell type. This

131 indicated that the influence of genotypic variation within our study population

132 outweighed the influence of cell type on DNAm patterns of CD4 and CD8 T cells in cord

133 blood. To further test this, we performed a silhouette analysis, with cell types as

134 clusters. Consistent with expectations, all cell types clustered relatively well, with the 135 exception of CD8 T cells, where cord CD8 T cells did not cluster well with adult CD8 T 136 cells (Figure S1).

138 Table 1: Summary of data sets used in this study

\section{Reinius}

Age

$\%$ female
Adult

0
Gervin

Cord

54.5 de Goede

Cord

71.4 
$\mathbf{N}$

$\begin{array}{llll}\text { CD8T } & 6 & 11 & 6 \\ \text { CD4T } & 6 & 11 & 7 \\ \text { B } & 6 & 11 & 7 \\ \text { NK } & 6 & 11 & 7 \\ \text { Granulocyte } & 18 & 11 & 7 \\ \text { Monocyte } & 6 & 11 & 7 \\ \text { nRBC } & 0 & 0 & 7\end{array}$

139
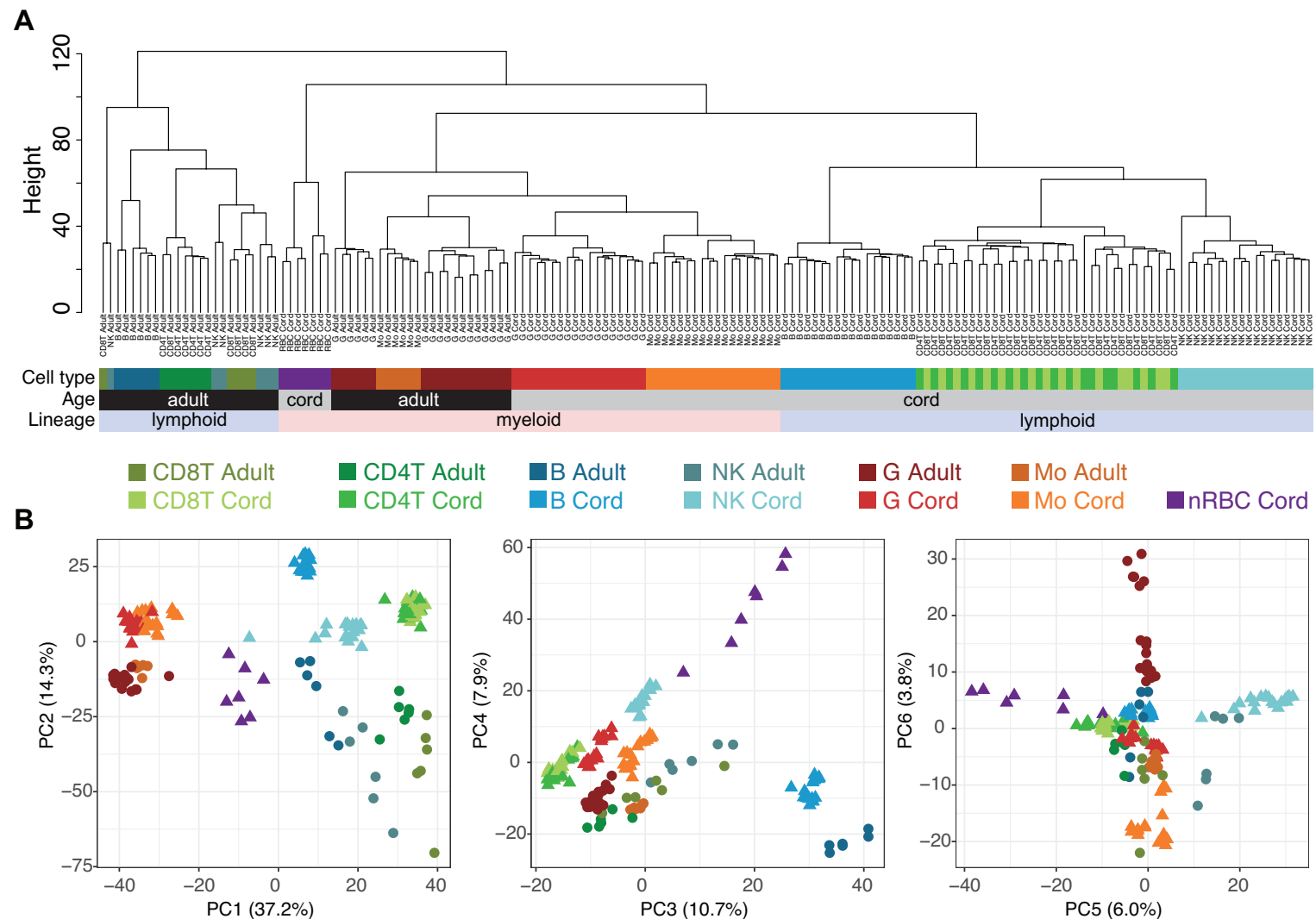

C

D
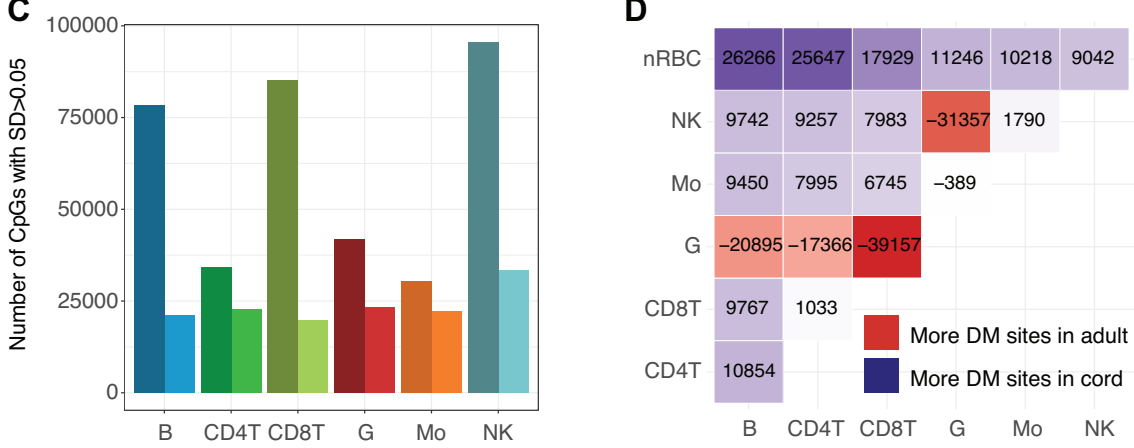
$143 \mathrm{n}=5$ for each adult cell type, $\mathrm{n}=18$ for cord B cells, CD4 T cells, granulocytes,

144 monocytes, and NK cells, $n=17$ for CD8 T cells, and $n=7$ for $n R B C s$. Samples clustered

145 first by lineage ( ink = myeloid, pale blue= lymphoid), then by age (black = adult, grey =

146 cord), and then by specific cell type (colour scale below). B) The first six principal components of the data set in $\mathrm{A}$, where circles are adult samples and triangles are cord blood samples, colours as above, and percent of variance indicated on the relevant axis. C) Number of sites in each cell type with an SD>0.05 in adult and cord cell types. See full counts of variable sites for all cell types and cell mixtures in Table S1. D) Heatmap showing number of sites that distinguish between each pair of cell types in adult versus cord data (adult $\mathrm{nRBC}$ values were set to zero). The red colour indicates that more sites distinguish these cell types in adult and purple indicates that more sites distinguish these cell types in cord.

Next, we used Principal Component Analysis (PCA) to determine how the patterns of variability in DNAm differed between cord and adult blood cell types. We first examined the first six principal components (PCs), accounting for more than $80 \%$ of the variance and which separate the different cell types. The first PC, accounting for $37 \%$ of the variance, separated the myeloid and lymphoid lineages (t-test $p<1 \times 10^{-16}$ ), with distinct clustering of cord and adult samples in the second PC (t-test $p<1 \times 10^{-16}$ ), accounting for $14 \%$ of the variance (Figure 1B). Myeloid cell types clustered more closely than

163 lymphoid cell types across PCs, perhaps reflecting a relative functional and lineage

164 proximity. These findings were consistent with the results of our hierarchical clustering

165 analysis. Next, we visually examined the spread of PC scores within a cell type, an

166 indication of how similar the samples within a cell type are to one another. Across both

167 of the top PCs, adult lymphoid cell types showed greater variability compared to myeloid 168 cell types (Figure 1B). The variability within adult lymphoid cells was also higher than

169 their corresponding cord blood cell types in these first PCs, which may reflect an

170 increasing proportion of differentiated effector and memory $\mathrm{T}$ and $\mathrm{B}$ cells due to antigen

171 exposure over lifespan.

172 To quantify the observed differences in variability between cord and adult blood 173 observed by PCA, we examined the number of variable sites within each cell type. We

174 hypothesized that a high number of variable sites in any particular cell type might make 175 it more difficult to identify tissue-specific sites, as variable sites within a cell type are 176 unlikely to be good cell type markers. Due to different sample numbers, we compared 177 the adult samples only to one sorted cord blood data set (de Goede), which have similar 
178 sample sizes ( $\mathrm{n}=6$ adult, $\mathrm{n}=7$ cord), but includes $\mathrm{nRBCs}$. We defined a variable probe 179 as having a beta value standard deviation greater than 0.05 (Table S1). Notably, nRBCs

180 exhibited a large number of variable probes $(77,888)$. All cell types showed more

181 variable sites in adult than in cord, and B cells, CD8 T cells, and NK cells showed

182 considerably more than CD4 T cells, monocytes and granulocytes. While overall, the 183 total numbers of variable sites were likely not high enough to influence the accuracy of

184 cell type prediction, higher variability in adult lymphoid cell types might be reflective of

185 inter-individual differences in adaptive immunity (Figure 1C, raw counts in Table S1).

186 We next determined whether cell type pairs were more or less difficult to distinguish

187 from one another in cord blood as compared to adult blood. To do this, we extracted the

188 number of differentially methylated probes within cord or adult whole blood using

189 pairwise comparisons for all cell types with a stringent nominal $p$ value of $1 \times 10^{-7}$. As

190 expected, we found different candidate cell type DNAm markers between the two ages,

191 but also noted important differences in the numbers of CpGs that can distinguish

192 between cord and adult blood cell types (Figure 1D). All pairs, except those involving

193 granulocytes, had more sites distinguishing pairs in cord than in adult samples. This

194 may be because cord samples were less variable than adult within a cell type as

195 observed in both PCA and the number of variable sites, making it easier to distinguish

196 one cell type from another.

197 To identify cell type specific DNAm differences between cord and adult blood, we

198 performed an epigenome-wide association study (EWAS) between cord and adult in the

199 six common cell types (CD4 T, CD8 T, NK, B, granulocyte and monocyte) and the two

200 cell mixtures (whole blood and mononuclear cells). As expected, a large number of

201 CpGs were differentially methylated between cord and adult blood at a $p$ value of $1 \times 10^{-7}$

202 and a mean DNAm difference of $10 \%$ (min 2989 for monocytes, max 9885 for

203 granulocytes, Figure 2A, Figure S2, Table S2). In the purified cell types, 588 CpGs were

204 differentially methylated between cord and adult samples in all six types, and 397 and

2052062 CpGs were lymphoid or myeloid specific, respectively (Figure 2A, full overlap in

206 Table S3). 
A

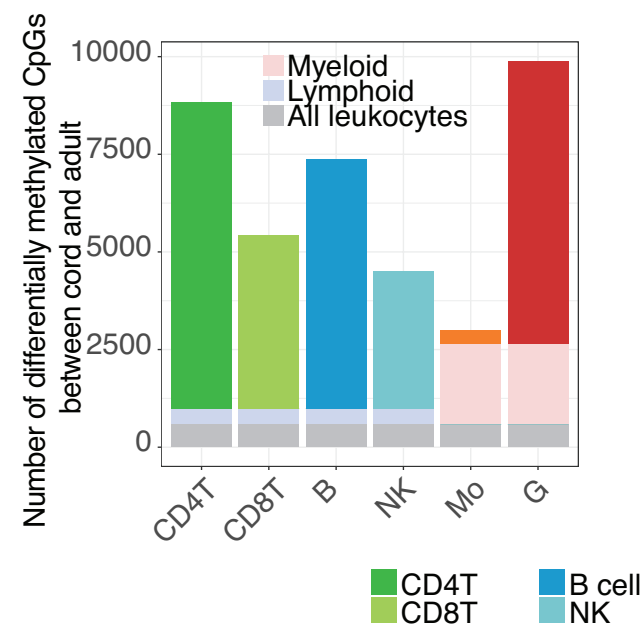

C

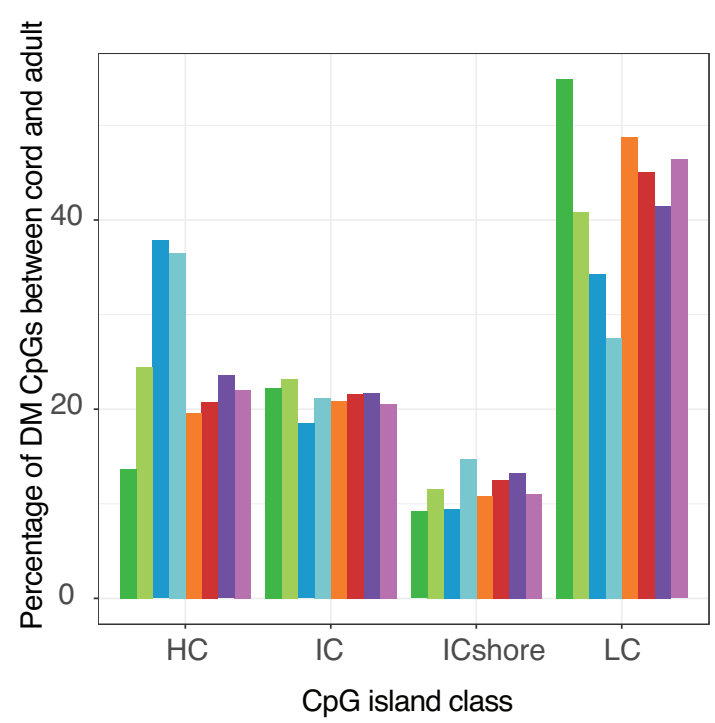

B

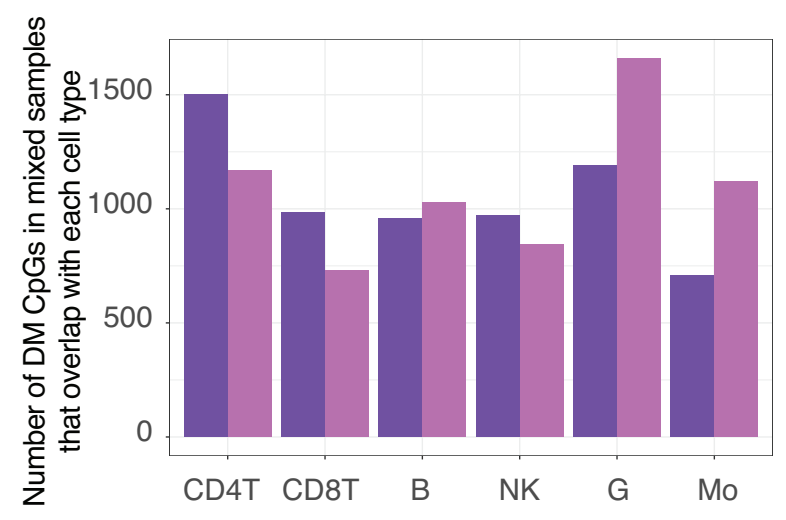
Monocytes
Granulocytes
Whole Blood

D

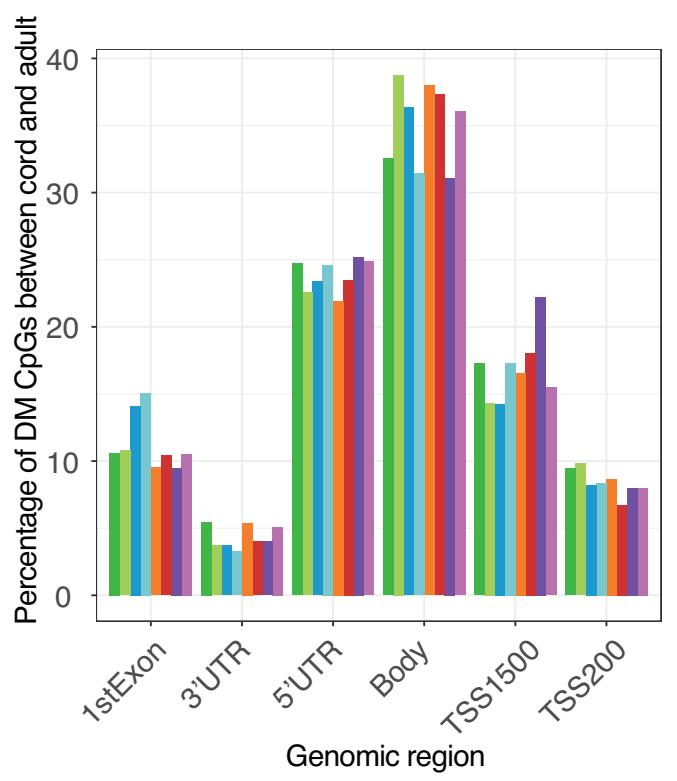

Figure 2. DNA methylation differences between cord and adult blood cells by cell type and genomic location. We performed an EWAS comparing cord to adult samples for each cell type, retaining sites with a $p$ value $<1 \times 10^{-7}$ and a mean DNA methylation difference $>10 \%$ (visualized in Figure S2). A) The number of significantly differentially methylated $\mathrm{CpGs}$ between cord and adult blood in the six cell types. Significant $\mathrm{CpGs}$ in all cell types are in grey $(\mathrm{N}=588)$, lymphoid specific $(\mathrm{N}=397)$ or myeloid specific

$(\mathrm{N}=2062) \mathrm{CpGs}$ are in pale blue or pink, respectively, and the remaining $\mathrm{CpGs}$ are in the colour of that cell type. Note that these might not all be unique to that cell type, but are neither common, nor specific to lymphoid or myeloid cells. Total pairwise overlap numbers are in Table S2. B) Number of CpGs in mixed tissues which were differentially methylated ( $\mathrm{N}=2558$ and $\mathrm{N}=1993$ in whole blood and mononuclear cells, respectively), and overlap with the differentially methylated sites in each cell type. The number of sites common across all cell types and cell mixtures ( $N=507$ out of 588 in grey in part $A$ ) was subtracted from the total number. C) Proportion of differentially methylated $\mathrm{CpGs}$ in each cell type and cell mixture that fall within each of the four $\mathrm{CpG}$ island classes $(\mathrm{HC}=$ high density, IC = intermediate density, ICshore $=$ CpG island shore LC=low density). $\mathbf{D}$ ) 
224 Proportion of differentially methylated CpGs in each cell type and cell mixture that fall

225 within five common genomic features. Sites not annotated to a specific region are not

226 shown.

228 In an effort to determine how much of these differences were due to genetic effects,

229 given that our cord and adult samples were not from the same individuals, we examined

230 the overlap with CpGs previously identified as being associated with genotype (mQTLs)

231 in cord blood in the ARIES data set (Table S3). ${ }^{8}$ We hypothesized that genetic effects

232 would be observed at highest proportion in those sites which were differentially

233 methylated between cord and adult samples in all cell types, as cross-tissue genetic

234 effects seem to be more frequent than tissue-specific genetic effects. ${ }^{26,27}$ The results

235 from these analyses revealed that between 9 and $11 \%$ of the myeloid- and lymphoid-

236 specific differentially methylated sites were currently reported mQTLs. In the individual

237 lymphoid cell types, 6-12\% of the differentially methylated CpGs between cord and adult

238 blood were cord blood mQTLs. Interestingly, the myeloid cell types showed a very

239 different pattern, where $18 \%$ of the differentially methylated CpGs in granulocytes and

$24079 \%$ in monocytes were cord blood mQTLs. This result was surprising, as it implies that

241 many of the differentially methylated CpGs between cord and adult blood in monocytes

242 might be mQTLs, despite the fact that it already had the smallest number of differentially

243 methylated sites among cell types. This could have interesting implications for future

244 assessment of genetic influences on cell type specific DNAm.

245 Next, we examined the differentially methylated sites between cord and adult in two

246 commonly used cell mixtures; whole blood, which contains all cell types and

247 granulocytes are by far the most prevalent and blood enriched for mononuclear cells,

248 which primarily removes granulocytes, leaving CD4T cells as the most prevalent cell

249 type. We hypothesized that the differences between cord and adult in these mixtures

250 would be influenced by the underlying cell proportions, meaning that differentially

251 methylated CpGs in each mixture would overlap most with the most prevalent cell type

252 in that mixture. This was indeed observed, with differentially methylated sites in

253 mononuclear cells overlapping most with those sites which were differentially

254 methylated in CD4 T cells and, and likewise whole blood sites overlapped most with

255 granulocyte sites (Figure 2B). 
256 Finally, we examined the genomic feature locations of the differentially methylated sites

257 between cord and adult in all cell types by mapping each CpG to CpG island class (HC:

258 high density CpG island, LC: low density CpG island, IC: intermediate density CpG

259 island) and genomic features. In NK and B cells, more CpGs mapped to high density

260 (HC) islands and less for low density (LC) islands. CD4 T cells showed the opposite

261 pattern. Overall the cell types were quite consistent in enrichment for CpG island status

262 (Figure 2C). Few differences between the cell types and mixtures were observed for 263 enrichment of the six genomic regions ( $1^{\text {st }}$ exon, 3'UTR, 5'UTR, gene body, TSS200, 264 and TSS1500) investigated (Figure 2D).

Probe type selection method and inclusion of $n R B C s$ influenced cell type prediction

267 accuracy in cord blood

268 Given that DNAm showed substantial differences between cord and adult blood in both 269 variability and at specific sites across the genome, we next identified which parts of the 270 deconvolution algorithm might be affecting the accuracy of predictions in cord blood. To 271 do this, we used a validation data set of 24 whole cord blood samples from which both 272 DNAm measurements and matched cell counts determined by flow cytometry were 273 available. First, we applied the existing deconvolution algorithm from the minfi package 274 using default settings to the test data using the adult references, and repeated the same 275 prediction using the cord references that included nRBCs. The results from these 276 analyses revealed moderate prediction of cord blood cell types using adult references, 277 and slightly improved predictions using the de Goede cord blood references, in 278 agreement with previous studies (Figure S3). ${ }^{20-22}$

279 We then hypothesized that the method for selecting sites to use in deconvolution could 280 influence its prediction accuracy, as the observed differences between cord and adult

281 DNAm mean that the method created for adult blood may be less effective on cord

282 blood. Several selection heuristics have been proposed and modified over the past few 283 years. ${ }^{28,29}$ The original method selected the top 50 probes that display higher and lower 284 DNAm in each cell type according to their effect size, for a total of 100 probes per cell 285 type. In cord blood, we replicated a previous finding that for monocytes in particular, this 
selection method chooses many sites that do not distinguish between monocytes and other cell types, as there are less than 20 monocyte markers that have higher DNAm in monocytes than other cell types (Figure S4). ${ }^{22}$ This means that in cord blood, forcing probe selection to include an equal number of higher and lower methylated probes would adversely affect monocyte prediction at the very least, which would in turn reduce the accuracy of prediction for the other cell types.

292 Next we examined the predictions of nRBCs, which can account for up to $25 \%$ of the 293 nucleated cell composition in cord blood, and possibly more of the DNAm signal due to 294 cell free DNA from nRBCs that had already extruded their nuclei. ${ }^{25}$ As previously 295 reported, nRBCs have a unique DNAm profile in cord blood, quite different from the 296 typical bimodal distribution of DNAm patterns in other cell types (Figure S5A). ${ }^{25}$ In 297 addition, not including nRBCs in the reference library, as occurs when using the Gervin 298 reference data set, violates one of the assumptions of the deconvolution method, which 299 is that all major cell types are represented in the reference set. ${ }^{18}$ Thus, we assessed the 300 impact of removing $\mathrm{nRBC}$ from our reference set. For each sample in the validation data set, we predicted cell type proportions with and without the nRBCs included. We then calculated percentage change in estimated proportion for each sample and found an uneven impact across cell types, with B cells (20\% mean, 50\% maximum), monocyte (10\% mean, $52 \%$ maximum) and NK (21\% mean, $62 \%$ maximum) cells having the largest percentage difference in predicted cell type caused by the removal of $\mathrm{nRBCs}$ from the reference set (Figure S5A). The magnitude of impact was related to both the abundance of cell type and similarity of DNAm profiles between cord blood cell types as shown by hierarchical clustering across discriminating probes used in the deconvolution (Figure S5B). This demonstrated how inclusion of $\mathrm{nRBCs}$, which displayed distinct

312 Using cord references, modified probe selection, and including $n R B C s$ resulted in 313 improved age-specific cell type prediction accuracies in whole blood data sets

314 Our results have shown that the difference in performance between adult and cord 315 blood were likely not due to any single main factor, but rather the compounding of 
316 multiple effects based on the unique properties of cord blood. By resolving the issues

317 identified above, we produced cord blood prediction performance that were more

318 comparable to previously reported adult whole blood deconvolution in our validation

319 data (B cell rho=0.73, CD4T rho=0.84, CD8T rho=0.40, gran rho=0.64, mono rho=0.53,

$320 \mathrm{NK}$ rho=0.67, nRBC rho=0.66, Figure 3A). ${ }^{19}$ All cell types showed good correlations,

321 though nRBCs and CD8 T cells seemed to be over-estimated across all samples. PCA

322 analysis shows that most of our predicted cell type proportions were significantly

323 associated with PC1 of cord blood DNAm, as expected (Figure 3B). Interestingly, the

324 signal for nRBCs, specific to cord blood, was only associated with PC2 and PC4,

325 signifying that the nRBC contribution to DNAm pattern in cord blood accounts for less

326 variance than the other cell types. 
A. Cord references, cord samples, optimized method
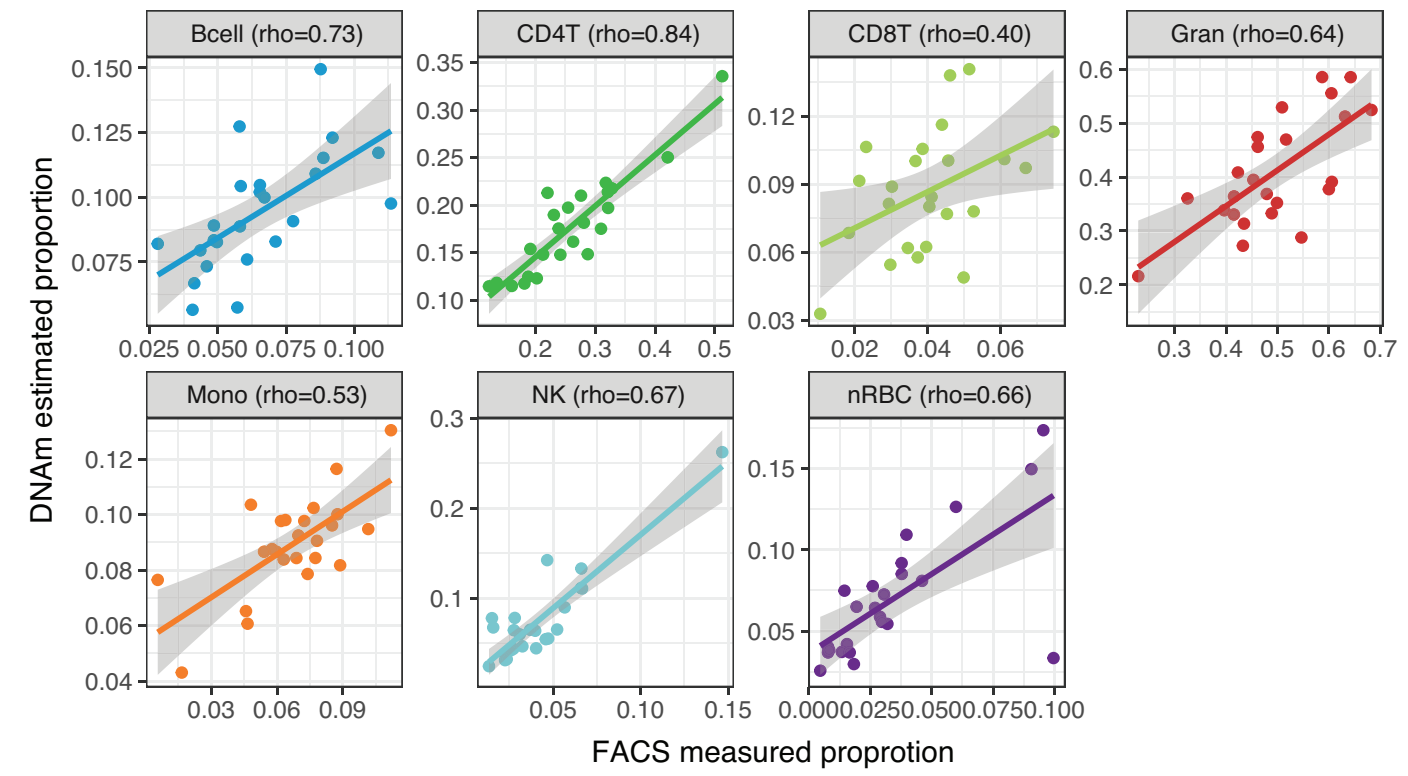

B.
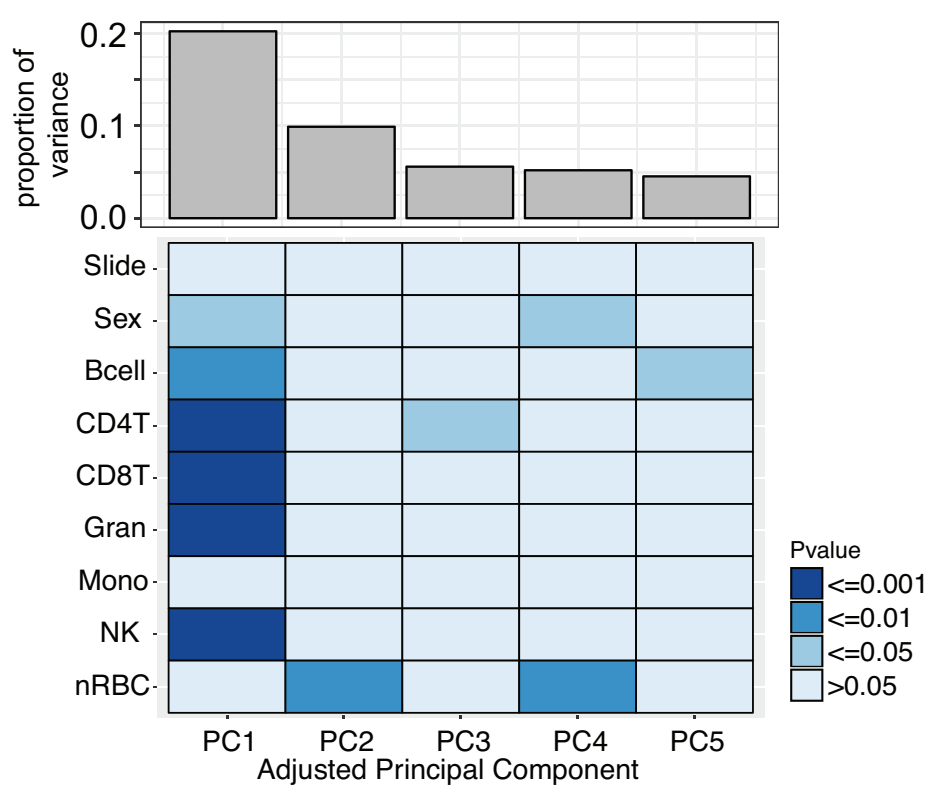

Figure 3: Including $\mathrm{nRBCs}$, altering the probe selection, and using cord references improved deconvolution accuracy in cord blood. A) flow cytometrybased cell counts ( $x$ axis) compared with DNAm predicted ( $y$ axis) enumeration for each of the seven cord blood cell types. Spearman's rho for each is shown. Coloured lines indicate regression line for each cell type, and shaded areas $95 \%$ confidence interval. B) PCA on 24 whole cord bloods shows associations between deconvolution-based cell counts and PC1, as expected.

336 To validate the modifications to the cord blood cell type prediction method, we applied

337 our method to an external data set of 191 cord blood samples with flow cytometry- 
338 based cell type enumerations from the Generation R cohort study. 10,20,30 Unfortunately, 339 this validation data set has not measured nRBCs, and so although we predicted nRBCs, 340 we were unable to validate predictions of this important cell type. However, the

341 prediction accuracy of the other cell types were generally higher than previously shown

342 (Figure 4). ${ }^{20}$ This indicates that the combination of using cord references and including

343 nRBCs combined with correct probe type selection for cord blood result in accurate

344 predictions across data generating platforms. 


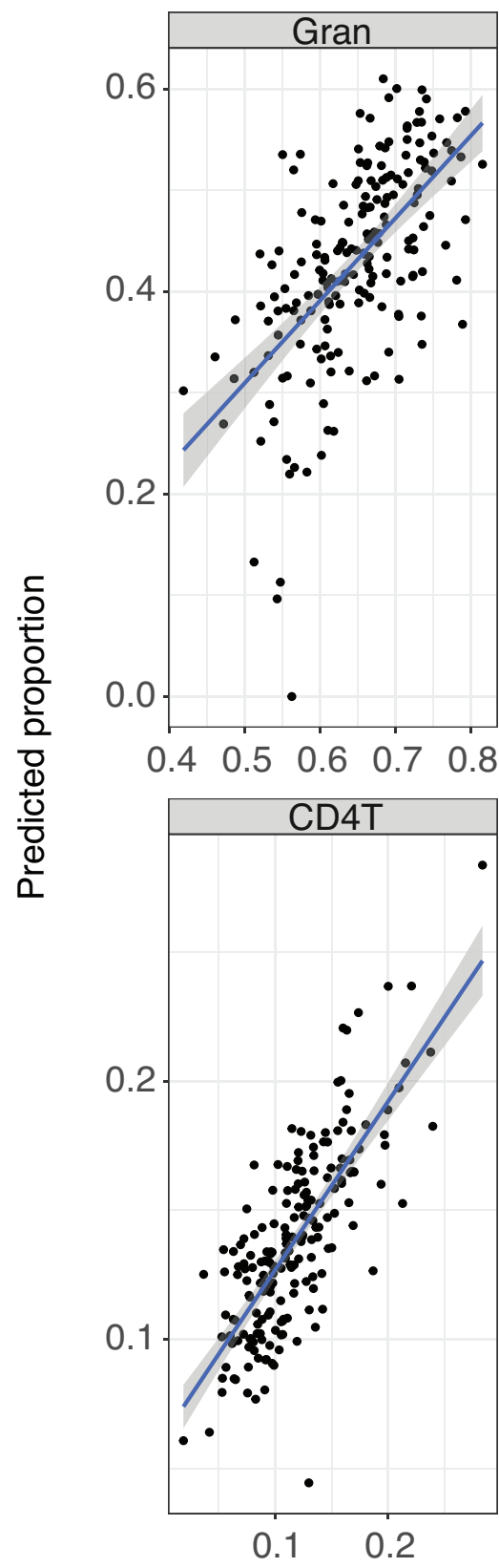

\section{Discussion}
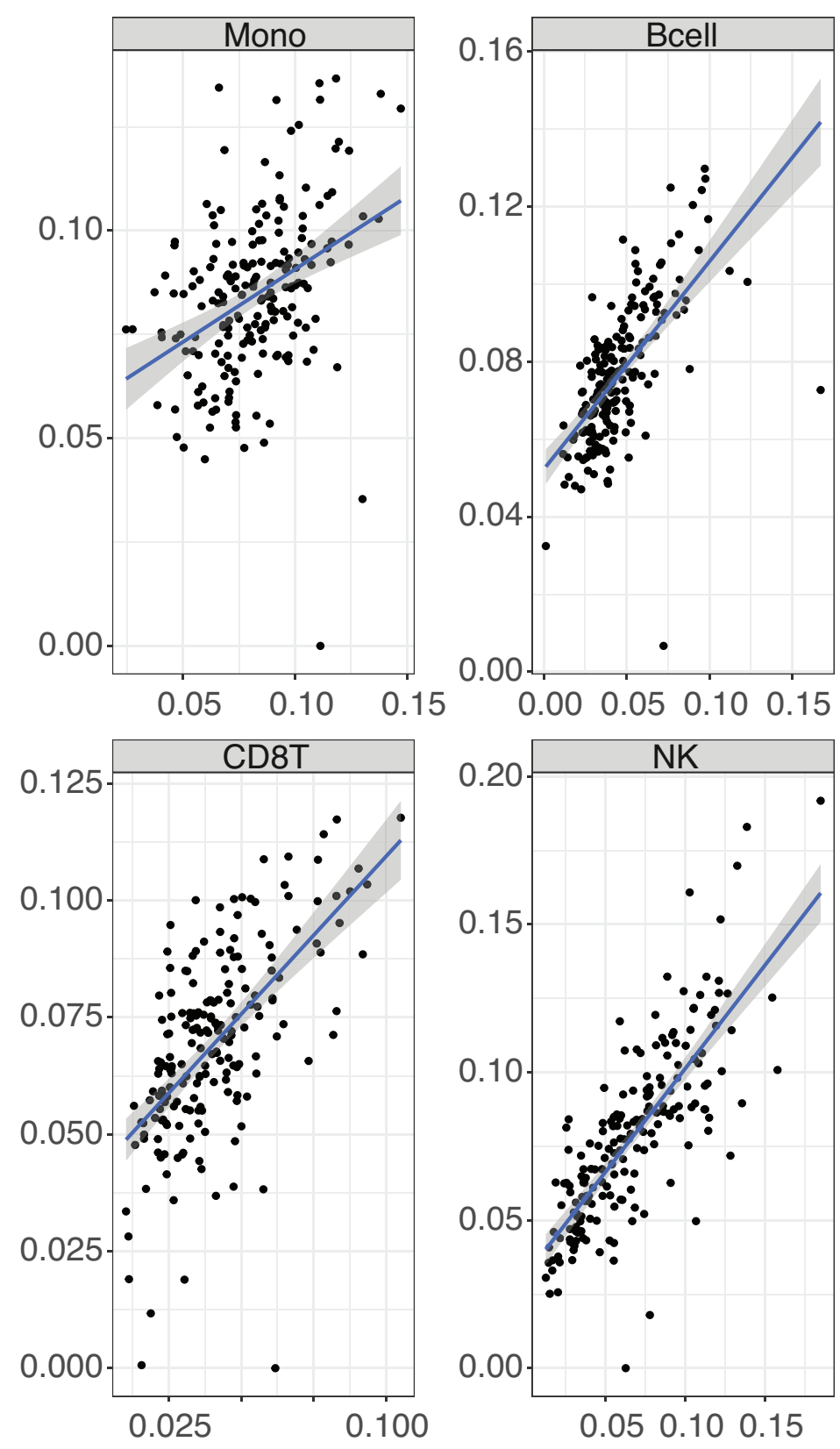

FACS measured proportion

Figure 4: Improved deconvolution resulted in improved prediction accuracy in an independent validation cohort of 191 cord blood samples. Blue line indicates linear regression line, and grey shading indicates $95 \%$ confidence interval. nRBCs were predicted using deconvolution but not measured using FACS and so are not shown.

Here, we have explored intrinsic biological differences in DNAm between cord and adult blood cell types. In addition to providing important insights into the fundamental 
355 developmental trajectories of DNAm, these analyses led to important adaptations to

356 deconvolution methods that are necessary for accurate predictions of cell type

357 composition in whole cord blood samples. It has been previously shown that DNAm is

358 highly variable with age, but age-related effects on individual cell types have not been

359 as well studied. ${ }^{2,24}$

360 At least two clear differences exist between cord and adult white blood cells that could 361 influence DNAm, and which might differ across cell types. The first is the impact of age 362 and immunological maturation of white blood cell types after birth. ${ }^{2,31,32}$ While not yet 363 documented, it was perhaps not surprising that DNAm patterns from cord and adult cell 364 types were visually distinct in both clustering and PCA analysis. However, the number 365 of variable sites within cell types was highly different between cord and adult samples.

366 Though all cell types had distinct PCA patterns between cord and adult, this was 367 accentuated in lymphoid than myeloid cells. This observation likely reflected a 368 predominant functional maturation of lymphoid cells post-natally, in contrast to myeloid 369 cells whose function matures predominantly earlier during fetal development. 10,31,32

370 Further, CD4 and CD8 T cells clustered very differently in cord blood than in adult 371 blood. In adults, CD4 and CD8 T cells clustered separately, whereas in cord blood they 372 clustered by individual rather than by cell type. These results suggested that neonatal 373 CD4 and CD8 T cells were more similar at the DNAm level, which may reflect a relative 374 lack of functional differentiation between these two cell lineages prior to antigen 375 exposure. This may also explain why CD8 T cells proportions were difficult to predict 376 accurately in cord blood here, as was also observed in a previous study. ${ }^{20}$ It is possible 377 to predict CD4 T cells more accurately due higher abundance, compared to CD8 T 378 cells, which are both hard to discriminate and of lesser abundance in cord blood. ${ }^{33}$ 379 Thus, a possible solution could be to combine these two cell types for prediction of cell 380 composition in cord blood samples.

381 Both variability and EWAS results demonstrated that DNAm differences between cord 382 and adult blood were distinct between cell types. Adult cell types were more variable 383 than cord, with B, CD8 T, and NK cells showing the largest differences. EWAS within 384 cell types identified thousands of DNAm differences between cord and adult, but the 385 specific number of differentially methylated CpGs varied across cell types, with 
386 granulocytes showing the most differentially methylated sites and monocytes the least.

387 This finding is unusual, given that in the PCA analysis, both granulocytes and

388 monocytes showed more similar broad DNAm patterns between cord and adult than

389 any of the lymphoid cell types. Additionally, there were many more sites that differed

390 between cord and adult and were common between the two myeloid cell types than

391 between the lymphoid cells. This could be a further indication that at least at the level of

392 DNAm, myeloid cell types were more similar to one another than the lymphoid cell

393 types, but it was also possible that the overlap appeared higher because there are only

394 two myeloid cell types, rather than the four lymphoid, and so the overlap may be higher

395 by chance.

396 Interestingly, in lymphoid cells approximately $10 \%$ of the estimated differences between

397 cord and adult overlapped cord blood mQTLs, suggesting a genetic influence on the

398 DNAm variation. In myeloid cells it was much higher, up to $79 \%$ in monocytes,

399 suggesting that most of the differences between cord and adult cell types were actually

400 genetic effects. This implies much less DNAm change in monocytes with age compared

401 to other cell types, which is also consistent with previous reports. ${ }^{34}$ Combined with the

402 finding that monocytes, uniquely of all cell types, do not have many probes that are

403 more methylated compared to the other cell types, monocytes seem to be quite different

404 from other cell types in terms of their changes in DNAm with age. This may partly

405 explain why, even after modifying the cord blood prediction, monocytes remain one of

406 the most difficult cell types to predict, with the worst correlation coefficient of any cell

407 type in the GenR data and the second worst in our validation data.

408 The second major difference between cord and adult blood that might impact

409 deconvolution is the presence of nRBCs. Due to their abundance and unusual DNAm

410 patterns, the presence of nRBCs influences DNAm pattern in cord blood, but to date

411 their impact on estimation of cell type proportions has been poorly understood. ${ }^{25,35} \mathrm{We}$

412 showed that omitting $\mathrm{nRBC}$ from the predictions reduced accuracy of predicting the

413 other cell types. This analysis documented how heavily the constrained projection

414 framework depends on a reference of each major cell type in the mixture. Eliminating

415 one cell type will reduce prediction performance, though it is not always clear which cell

416 type will be allocated to make up for one that is missing, and nRBCs may be particularly 
417 prone to this due to their unusual DNAm pattern. ${ }^{18}$ In addition, we note that while the 418 predicted $\mathrm{nRBC}$ proportions were well correlated with the measured proportions, these 419 were all scaled proportionally higher. One possible explanation is that the amount of $420 \mathrm{nRBC}$ DNA in a sample is not all contained within nRBCs and thus not reflected in cell 421 counts. As these samples were derived from term births, red blood cells are in the 422 process of extruding their nuclei, potentially leaving acellular DNA in the extracellular 423 material which would then be collected along with the nuclear DNA from intact cells ${ }^{36}$. 424 Such a process could explain the difference observed, as the deconvolution method is 425 predicting the proportion of nRBC DNA, not cells. Given the high correlation, we are 426 confident that the predictions were accurate for use as corrections, although using the 427 magnitudes of predicted $\mathrm{nRBC}$ counts as an outcome should be done with caution. Of 428 additional interest, although nRBCs are common in cord blood, they are not unheard of 429 in adults, with substantial amounts having been reported in anemias, some leukemias, 430 and some cardiac conditions. ${ }^{37}$ In those cases, prediction of cell type composition by

431 DNAm using adult references may demonstrate reduced accuracy, as shown in our 432 experiment removing nRBCs in cord blood.

433 Putting all of our findings into a bigger perspective, we believe that reference-based 434 prediction techniques are currently the best option for dealing with inter-individual 435 differences in cell type proportions where cell counts are not available. In this case, our 436 findings have shown that biological differences are associated with prediction accuracy, 437 but this approach is not without limitations. First, our reference dataset is based on cell 438 purification using FACS. This technology discriminates based on cell surface markers 439 and does not distinguish between subpopulations within a particular cell type, which 440 may also differ across development. ${ }^{9}$ Second, cell counts for our validation data were 441 quantified using a combination of flow cytometry and the complete blood count. This 442 combination of methods runs the risk of compounding errors from the two methods and 443 thus decreasing accuracy. However, given that we were able to show high accuracy of 444 prediction on samples from two independent data sets, we believe that these counts 445 were sufficiently accurate for correction in EWAS studies. Finally, the adult and cord 446 data came from different data sets and different individuals, which means that both 447 genetic differences and batch effects might inflate our estimate of age-specific 
448 differences. To account for genetic differences, we assessed mQTL enrichment of our

449 age-specific findings as described. Batch effects are more difficult, as they are

450 confounded by age, and therefore not possible to specifically remove. However, since

451 there is no expectation that batch effects would be cell-type specific, the comparisons

452 between cell types should be equally affected by batch effects, and thus not bias the

453 interpretation of the findings.

\section{Conclusions}

456 The exciting potential of epigenetic profiling of cord blood as a marker of in utero

457 environmental exposure should be balanced by an understanding of the unique

458 properties of that tissue. Based on our results, it is clear that leukocyte of different

459 lineages mature differently in utero and after birth resulting in different DNAm between

460 cord and adult cell types. These appeared to be primarily driven by lymphocytes, which

461 have very similar DNAm profiles in cord blood compared to adults, mirroring their

462 acquisition of immunological memory postnatally upon antigen exposure. These findings

463 suggest an important functional role of DNAm in immune cell maturation during

464 development, and indicate why DNAm-based tools that are generated in adults should

465 be applied to other ages like cord blood with care.

\section{Methods}

468 Sample collection

469 Sorted and validation cord blood samples collected at UBC were collected from term

470 elective caesarian deliveries at BC Women's Hospital. All mothers gave written

471 informed consent, and protocols were approved by University of British Columbia

472 Children's \& Women's Research Ethics Board (certificate numbers H07-02681 and

473 H04-70488).

474 Purification of cord blood reference panel

475 Cord blood cell types were purified as previously published. ${ }^{25}$ Briefly, we applied seven 476 whole cord blood samples to Lymphoprep (StemCell Technologies Inc., BC, Canada) 
477 density gradient to separate granulocytes from mononuclear cells. Granulocytes were

478 further separated from non-nucleated red blood cells by density gradient. The

479 mononuclear fraction (which include nRBCs) was separated into constituent cell types

480 using a stringent flow cytometry gating strategy, as described previously ${ }^{25}$ on a

481 FACSArialll (Becton Dickinson), generating purified populations of monocytes (CD3-,

482 CD19-, CD235-, CD14+), CD4 T cells (CD14-, CD19-, CD235-, CD3+, CD4+), CD8 T

483 cells (CD14-, CD19-, CD235-, CD3+, CD8+), NK cells (CD3-, CD19-, CD235-, CD14-,

484 CD56+), B cells (CD3-, CD14-, CD235-, CD19+), and nucleated red blood cells (CD3-,

485 CD14-, CD19-, CD235+, CD71+).

486 Quantification of cell type proportions in whole cord blood validation samples

487 In addition to the sorted cord blood cell types, we collected twenty-four cord blood

488 samples for validation. A small aliquot of each sample was sent to the BC Children's

489 Hospital hematology lab for complete blood count with differential (CBC). A second

490 aliquot was prepared as the reference samples above, with the same markers and

491 antibodies, after lysis of red blood cells using BD FACS Lysing Solution (BD

492 Bioscience). Final cell counts for nRBCs, granulocytes, monocytes and lymphocyte

493 subsets were determined combining the CBC and flow cytometry data. CBC provided

494 nRBC, monocyte, and granulocyte cells counts, as well as total lymphocytes. We then

495 scaled these counts to total 1, and calculated lymphocyte subsets (relative proportions

496 of B, NK, CD4T, and CD8T cells) by multiplying the total lymphocyte proportion by the

497 relative proportions of lymphocyte subtypes measured by flow cytometry.

498 Generation of DNAm data

499 DNA from sorted reference and whole validation cord blood samples was isolated using

500 a Qiagen DNAeasy DNA isolation kit (Qiagen, USA). 750ng of isolated DNA was

501 subjected to bisulfite conversion using the Zymo EZDNA bisulfite conversion kit (Zymo

502 Research, USA), then applied to Illumina 450k microarrays per manufacturer's

503 instructions. Raw data was imported into Illumina Genome Studio (Illumina, USA) for

504 background subtraction and colour correction, then exported into R statistical software

505 for analysis. Reference and validation data were processed and applied to the arrays in

506 separate batches to simulate typical applications. 
507 DNAm preprocessing included removing probes for high detection $p$-value (>0.01), low

508 bead coverage (<3), sex chromosomes, cross hybridizing probes, and SNP probes.

509 Next, we applied Noob to correct for background and BMIQ for probe type

510 normalization. ${ }^{38,39}$ Finally, we applied batch correction using ComBat, accounting for

511 chip variability while explicitly protecting cell type. ${ }^{40,41}$ The same protocol was used to

512 normalize the FACS-sorted cord blood data from our study and another study along with

513 the adult sorted data. ${ }^{3,20,25}$ Validation data from Generation R data was generated as

514 previously described. ${ }^{20,30,42}$ For this study, we obtained it as IDAT files and normalized it

515 using the same steps described above.

516 Comparison of cord and adult samples

517 The dendrogram was generated using complete linkage of a Euclidean distance matrix

518 of samples based on methylation beta values including all three of these FACS-sorted

519 cell type data sets, with samples coloured by cell type, age, and myeloid vs lymphoid

520 lineage. Silhouette analysis used the same distance matrix, clustered by cell type. We

521 performed PCA on the same data set using the prcomp function in R. For the number of

522 variable sites, we used only the adult data and our sorted data with similar N, counted

523 the number of sites with SD > 0.05 in beta value in each cell type by each age. To

524 assess pairwise differences between cell types within adult and cord blood, we also

525 used only our sorted cord blood data set with the adult data, and performed a two-group

526 t-test on methylation m-values to determine the number of differentially methylated

527 probes between each pair, using a nominal $p$ value of $1 \times 10^{-7}$. As DNAm beta values are

528 heteroscedastic, $\mathrm{M}$ values are a log transformation of beta values that avoids the typical

529 statistical problems with heteroscedasticity.

530 We calculated the number of sites which discriminated between cell types and were

531 higher- or lower- methylated in that cell type compared to others by first ranking all sites

532 by $p$ value calculated from a two sided t-test comparing that cell type to all other cell

533 types. Next, we took the top 50 sites that had a mean DNAm value higher in that cell

534 type than others, and the top 50 with a lower mean DNAm value, and plotted the

535 magnitude of mean DNAm difference between that cell type and the other cell types.

536 Epigenome-wide association study comparing cord to adult white blood cells 
537 EWAS analysis was performed on the adult and our sorted cord blood data sets. ${ }^{3,25}$ We

538 applied the R package limma with a categorical variable of cord vs adult and no other

539 covariates to normalized data, using a $p$ value cutoff of $1 \times 10^{-7}$ and a mean absolute

540 beta value difference of 0.1 to define a significant CpGs.

541 Cell type prediction

542 For prediction of cell type proportions in cord blood, we applied the constrained

543 projection quadratic programming (CP/QP) algorithm developed by Houseman et al.,

544 Houseman:2012km as implemented in the minfi package without modification, and

545 using either adult or cord reference libraries. ${ }^{3,5,18,25,29}$ We then quantified the sensitivity

546 of our procedure by comparing estimated proportions on the same set of samples

547 depending on whether or not a nRBC profile was available, and after optimized

548 preprocessing and feature selection. Finally, we performed deconvolution again using

549 the cord blood references and defining the sites used in deconvolution as the sites with

550 the top $f$ statistic regardless of direction of change on both our validation and the

551 Generation R data. Accuracy of deconvolution estimates with cell counts was measured

552 with Spearman's Rho in all cases.

553

554 Declarations

555 Ethics approval and consent to participate

556 The study protocol was approved by the Medical Ethical Committee of the Erasmus

557 Medical Centre, Rotterdam. Written informed consent was obtained for all participants.

Consent for publication

560 Not applicable

Availability of data and material

563 Reference cord and adult data and used in this study is available on GEO (GSE35069,

564 GSE82084). Validation samples that were internally generated are also available

565 (GEO\# TBD), and Generation R data may be available upon request from the study

566 coordinators. Full code for figures is available on GitHub

567 (https://github.com/megjones/Rotterdam_code/blob/master/CordvsAdult_figures), and

568 modified code for cord deconvolution is available

569 https://github.com/megjones/Rotterdam_code/blob/master/Initial_deconvolution_edited.

$570 \mathrm{R}$ 
Competing interests

573 The authors declare that they have no competing interests.

Funding UBC: 450k data was funded by the Sunny Hill BC Leadership Chair in Child Development to MSK. GenR: 450k data was funded by a grant from the Netherlands Genomics Initiative (NGI)/Netherlands Organisation for Scientific Research (NWO) Netherlands Consortium for Healthy Aging (NCHA; project nr. 050-060-810), by funds from the Genetic Laboratory of the Department of Internal Medicine, Erasmus MC, and by a grant from the National Institute of Child and Human Development (R01HD068437). JFF has received funding from the European Union's Horizon 2020 research and innovation programme under grant agreement No 633595 (DynaHEALTH). This project received funding from the European Union's Horizon 2020 research and innovation programme (733206, LIFECYCLE). LD received funding from the co-funded programme ERA-Net on Biomarkers for Nutrition and Health (ERA HDHL) (ALPHABET project, Horizon 2020 (grant agreement no 696295; 2017), ZonMW The Netherlands (no 529051014; 2017)). National Health and Medical Research Council (NHMRC) Senior Research Fellowship (GNT1117687) to MvZ.

\section{Authors' contributions}

MJJ conceived the study, performed data analysis, and drafted the manuscript. LD performed data analysis and helped draft the manuscript. HRR collected reference and validation cord blood samples. OdG performed FACS to generate reference samples. JLM and AMM ran the 450k arrays for the de Goede references and internal validation samples. KG, RN, and RL advised on study design and data analysis. LD, MCvZ, and HAM generated FACS data for the GenR cohort. WPR, DCK, JFF, PLM, and SM helped conceive the study, advised on study design, and helped edit the manuscript. MSK helped conceive the study, oversaw the study, and helped edit the manuscript. All

\section{Acknowledgements}

604 The general design of the Generation R Study is made possible by financial support 605 from the Erasmus Medical Center, Rotterdam, the Erasmus University Rotterdam, the 606 Netherlands Organization for Health Research and Development and the Ministry of 607 Health, Welfare and Sport. The Generation R Study is conducted by the Erasmus 608 Medical Center in close collaboration with the School of Law and Faculty of Social 609 Sciences of the Erasmus University Rotterdam, the Municipal Health Service Rotterdam 610 area, Rotterdam, the Rotterdam Homecare Foundation, Rotterdam and the Stichting 611 Trombosedienst \& Artsenlaboratorium Rijnmond (STAR-MDC), Rotterdam. We 612 gratefully acknowledge the contribution of children and parents, general practitioners, 613 hospitals, midwives and pharmacies in Rotterdam.

614 The generation and management of the Illumina 450K methylation array data (EWAS 615 data) for the Generation R Study was executed by the Human Genotyping Facility of the 
616 Genetic Laboratory of the Department of Internal Medicine, Erasmus MC, the

617 Netherlands. We thank Mr. Michael Verbiest, Ms. Mila Jhamai, Ms. Sarah Higgins, Mr.

618 Marijn Verkerk and Dr. Lisette Stolk for their help in creating the EWAS database.

\section{References}

1. Smith, Z. D. \& Meissner, A. DNA methylation: roles in mammalian development. Nat Rev Genet 14, 204-220 (2013).

2. Jones, M. J., Goodman, S. J. \& Kobor, M. S. DNA methylation and healthy human aging. Aging Cell 14, 924-932 (2015).

3. Reinius, L. E. et al. Differential DNA methylation in purified human blood cells: implications for cell lineage and studies on disease susceptibility. PLOS ONE 7, e41361 (2012).

4. Ziller, M. J. et al. Charting a dynamic DNA methylation landscape of the human genome. Nature 500, 477-481 (2013).

5. Jaffe, A. E. \& Irizarry, R. A. Accounting for cellular heterogeneity is critical in epigenome-wide association studies. Genome Biol 15, R31 (2014).

6. Farré, P. et al. Concordant and discordant DNA methylation signatures of aging in human blood and brain. Epigenetics Chromatin 8, 19 (2015).

7. Joubert, B. R. et al. DNA Methylation in Newborns and Maternal Smoking in Pregnancy: Genome-wide Consortium Meta-analysis. Am J Hum Genet 98, 680696 (2016).

8. Gaunt, T. R. et al. Systematic identification of genetic influences on methylation across the human life course. Genome Biol 17, 61 (2016).

9. Kan, B., Razzaghian, H. R. \& Lavoie, P. M. An Immunological Perspective on Neonatal Sepsis. Trends in Molecular Medicine 22, 290-302 (2016).

10. van den Heuvel, D. et al. Effects of nongenetic factors on immune cell dynamics in early childhood: The Generation R Study. Journal of Allergy and Clinical Immunology 139, 1923-1934.e17 (2017).

11. Holt, P. G. \& Jones, C. A. The development of the immune system during pregnancy and early life. Allergy 55, 688-697 (2000).

12. Quinello, C. et al. Phenotypic differences in leucocyte populations among healthy preterm and full-term newborns. Scand. J. Immunol. 80, 57-70 (2014).

13. Kollmann, T. R., Levy, O., Montgomery, R. R. \& Goriely, S. Innate immune function by Toll-like receptors: distinct responses in newborns and the elderly. Immunity 37, 771-783 (2012).

14. Netea, M. G. Training innate immunity: the changing concept of immunological memory in innate host defence. Eur. J. Clin. Invest. 43, 881-884 (2013).

15. Shen-Orr, S. S. et al. Cell type-specific gene expression differences in complex tissues. Nat Methods 7, 287-289 (2010).

16. Lam, L. L. et al. Factors underlying variable DNA methylation in a human community cohort. Proc Natl Acad Sci USA 109 Suppl 2, 17253-17260 (2012).

17. Liu, Y., Balaraman, Y., Wang, G., Nephew, K. P. \& Zhou, F. C. Alcohol exposure alters DNA methylation profiles in mouse embryos at early neurulation.

660 epigenetics 4, 500-511 (2009). 
661

662

18. Houseman, E. A. et al. DNA methylation arrays as surrogate measures of cell mixture distribution. BMC Bioinformatics 13, 86 (2012).

19. Koestler, D. C. et al. Blood-based profiles of DNA methylation predict the underlying distribution of cell types: a validation analysis. Epigenetics : official journal of the DNA Methylation Society 8, 816-826 (2013).

20. Gervin, K. et al. Cell type specific DNA methylation in cord blood: a 450Kreference data set and cell count-based validation of estimated cell type composition. Epigenetics : official journal of the DNA Methylation Society 0 (2016). doi:10.1080/15592294.2016.1214782

21. Yousefi, P. et al. Estimation of blood cellular heterogeneity in newborns and children for epigenome-wide association studies. Environ. Mol. Mutagen. 56, 751758 (2015).

22. Bakulski, K. M. et al. DNA methylation of cord blood cell types: Applications for mixed cell birth studies. Epigenetics : official journal of the DNA Methylation Society 1-9 (2016). doi:10.1080/15592294.2016.1161875

23. Koch, C. M. et al. Specific age-associated DNA methylation changes in human dermal fibroblasts. PLoS ONE 6, e16679 (2011).

24. Horvath, S. DNA methylation age of human tissues and cell types. Genome Biol 14, R115 (2013).

25. de Goede, O. M. et al. Nucleated red blood cells impact DNA methylation and expression analyses of cord blood hematopoietic cells. Clin Epigenetics 7, 95 (2015).

26. Lin, D. et al. Characterization of cross-tissue genetic-epigenetic effects and their patterns in schizophrenia. Genome Med 10, 13 (2018).

27. Smith, A. K. et al. Methylation quantitative trait loci (meQTLs) are consistently detected across ancestry, developmental stage, and tissue type. BMC Genomics 15, 145 (2014).

28. Koestler, D. C. et al. Improving cell mixture deconvolution by identifying optimal DNA methylation libraries (IDOL). BMC Bioinformatics 17, 120 (2016).

29. Aryee, M. J. et al. Minfi: a flexible and comprehensive Bioconductor package for the analysis of Infinium DNA methylation microarrays. Bioinformatics 30, 13631369 (2014).

30. Kruithof, C. J. et al. The Generation R Study: Biobank update 2015. Eur J Epidemiol 29, 911-927 (2014).

31. de Jong, E., Strunk, T., Burgner, D., Lavoie, P. M. \& Currie, A. The phenotype and function of preterm infant monocytes: implications for susceptibility to infection. Journal of Leukocyte Biology 102, 645-656 (2017).

32. Sharma, A. A. et al. Hierarchical Maturation of Innate Immune Defences in Very Preterm Neonates. NEO 106, 1-9 (2014).

33. Comans-Bitter, W. M. et al. Immunophenotyping of blood lymphocytes in childhood. Reference values for lymphocyte subpopulations. J. Pediatr. 130, 388393 (1997).

34. Ecker, S. et al. Genome-wide analysis of differential transcriptional and epigenetic variability across human immune cell types. Genome Biol 18, 18 (2017). 
35. de Goede, O. M., Lavoie, P. M. \& Robinson, W. P. Characterizing the hypomethylated DNA methylation profile of nucleated red blood cells from cord blood. Epigenomics 8, 1481-1494 (2016).

36. Hebiguchi, M. et al. Dynamics of human erythroblast enucleation. Int J Hematol 88, 498-507 (2008).

37. Schwartz, S. O. \& Stansbury, F. Significance of nucleated red blood cells in peripheral blood; analysis of 1,496 cases. J Am Med Assoc 154, 1339-1340 (1954).

38. Triche, T. J., Weisenberger, D. J., Van Den Berg, D., Laird, P. W. \& Siegmund, K. D. Low-level processing of Illumina Infinium DNA Methylation BeadArrays. Nucleic Acids Research 41, e90-e90 (2013).

39. Teschendorff, A. E. et al. A beta-mixture quantile normalization method for correcting probe design bias in Illumina Infinium $450 \mathrm{k}$ DNA methylation data. Bioinformatics 29, 189-196 (2013).

40. Johnson, W. E., Li, C. \& Rabinovic, A. Adjusting batch effects in microarray expression data using empirical Bayes methods. Biostatistics 8, 118-127 (2007).

41. Chen, C. et al. Removing batch effects in analysis of expression microarray data: an evaluation of six batch adjustment methods. PLoS ONE 6, e17238 (2011).

42. Kooijman, M. N. et al. The Generation R Study: design and cohort update 2017. Eur J Epidemiol 31, 1243-1264 (2017).

\section{Supplementary figures and tables}




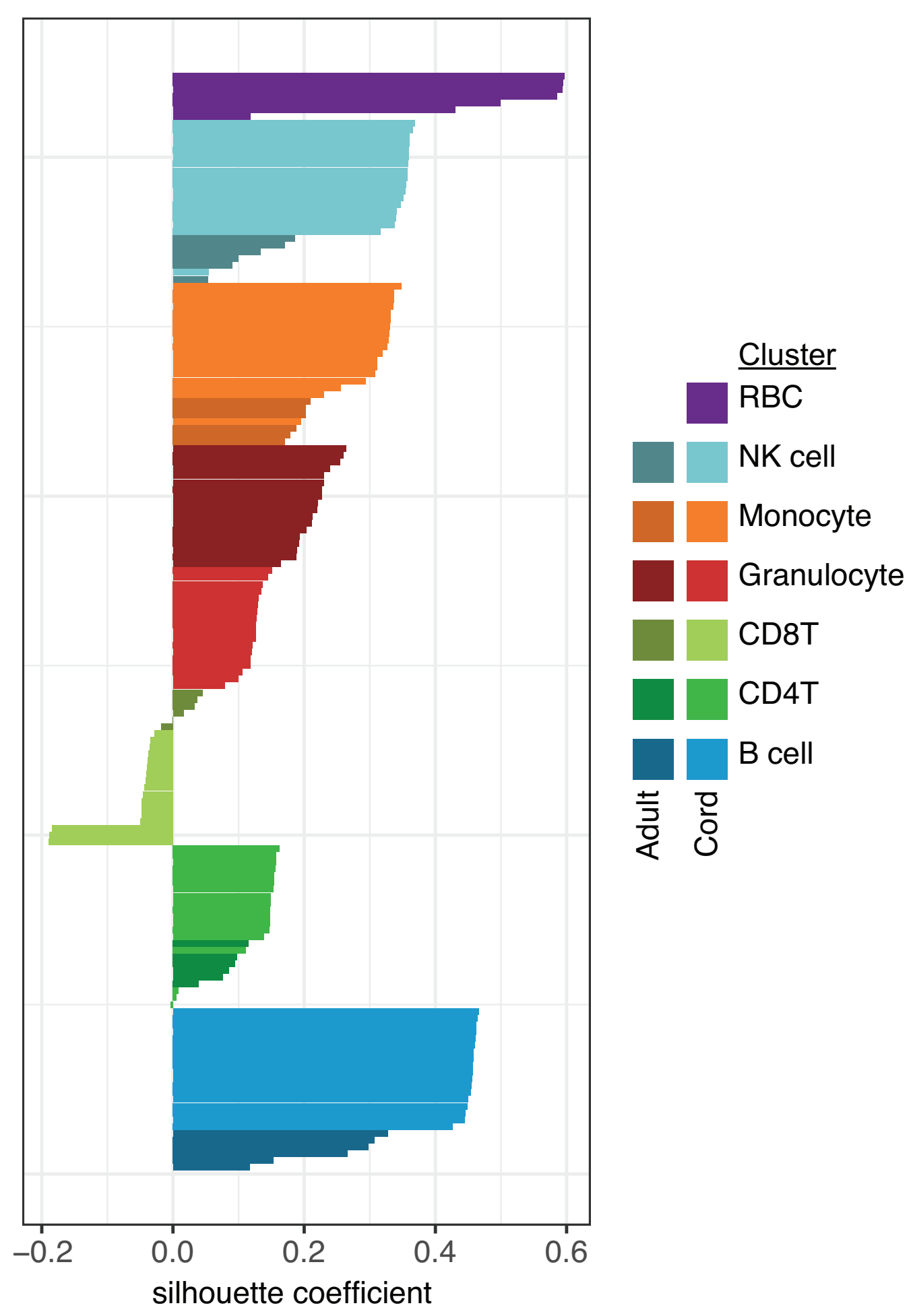

Figure S1: DNA methylation patterns of leukocyte cell types cluster together.

730 Silhouette plot based on DNAm data show 7 clusters. Cord and adult samples are

731 indicated in the same colour, but different shades (dark for adult and light for cord blood 732 data). 

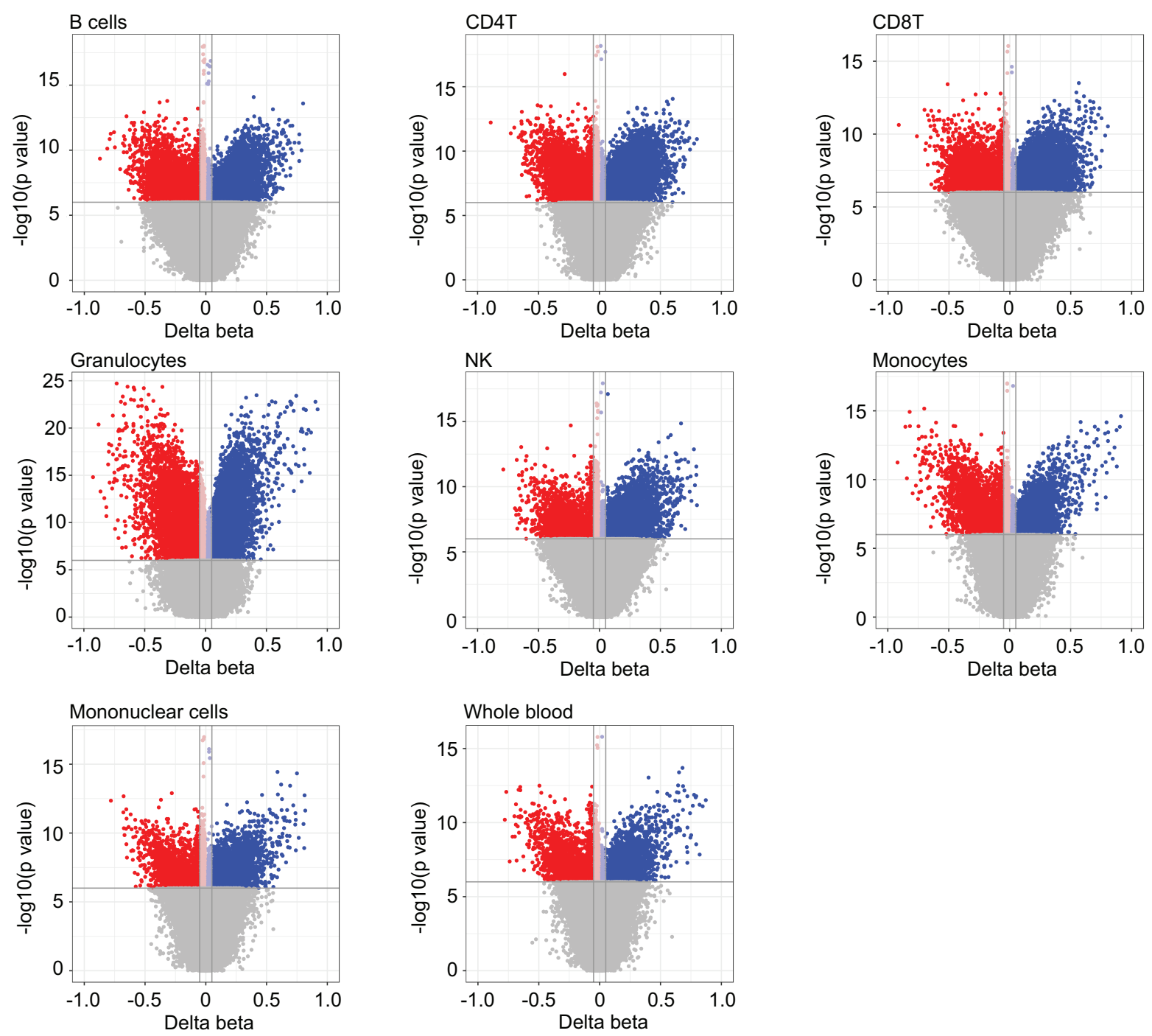

Figure S2: EWAS analysis shows large differences between cord and adult in all cell types. Volcano plots for each component cell type and two cell mixtures (whole blood and mononuclear cells). Sites in grey did not meet the $1 \times 10^{-7} p$ value cutoff. Sites in light red and light blue did not meet the absolute beta value difference of 0.1 . 
A. Adult references, cord samples

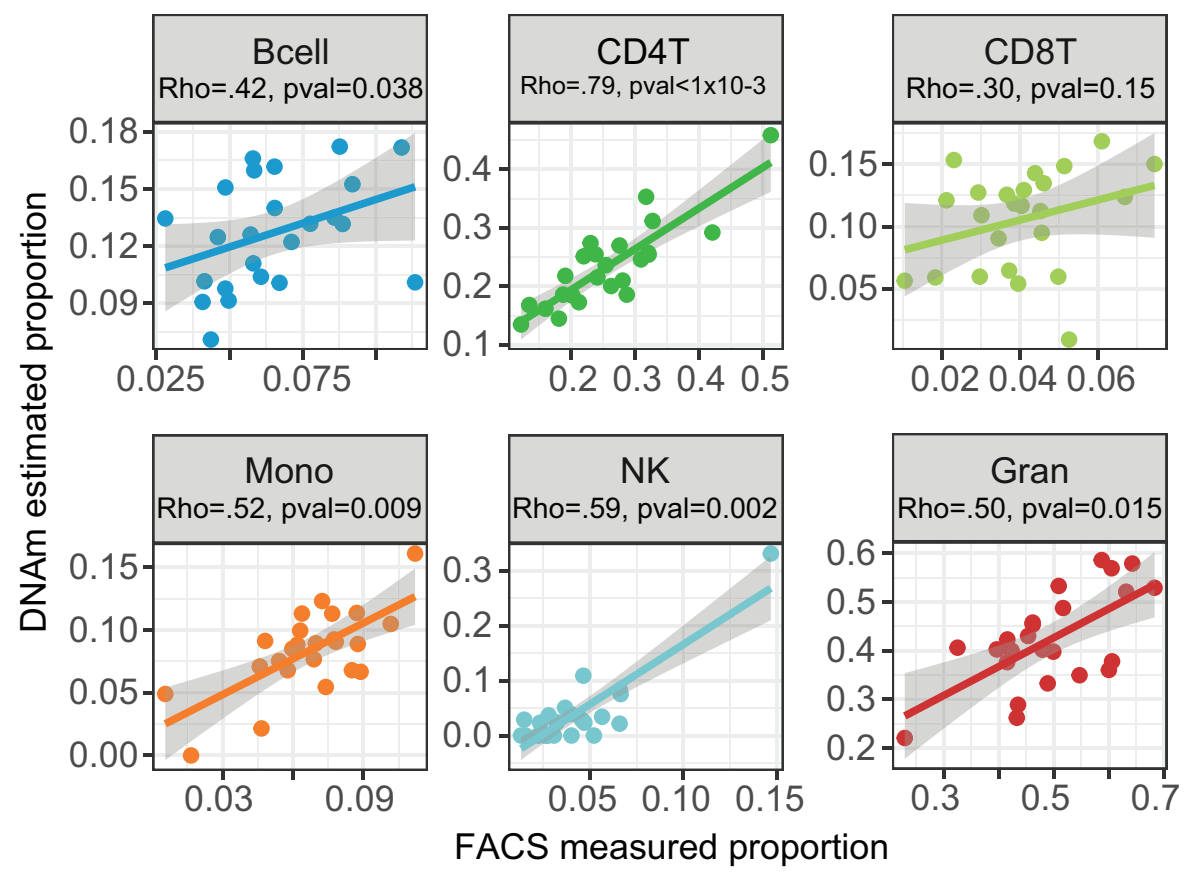

B. Cord references, cord samples

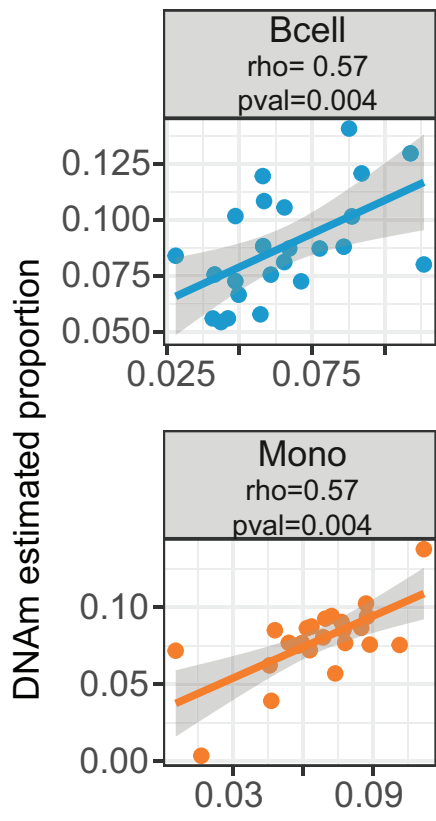

739

740

741

742

743

744
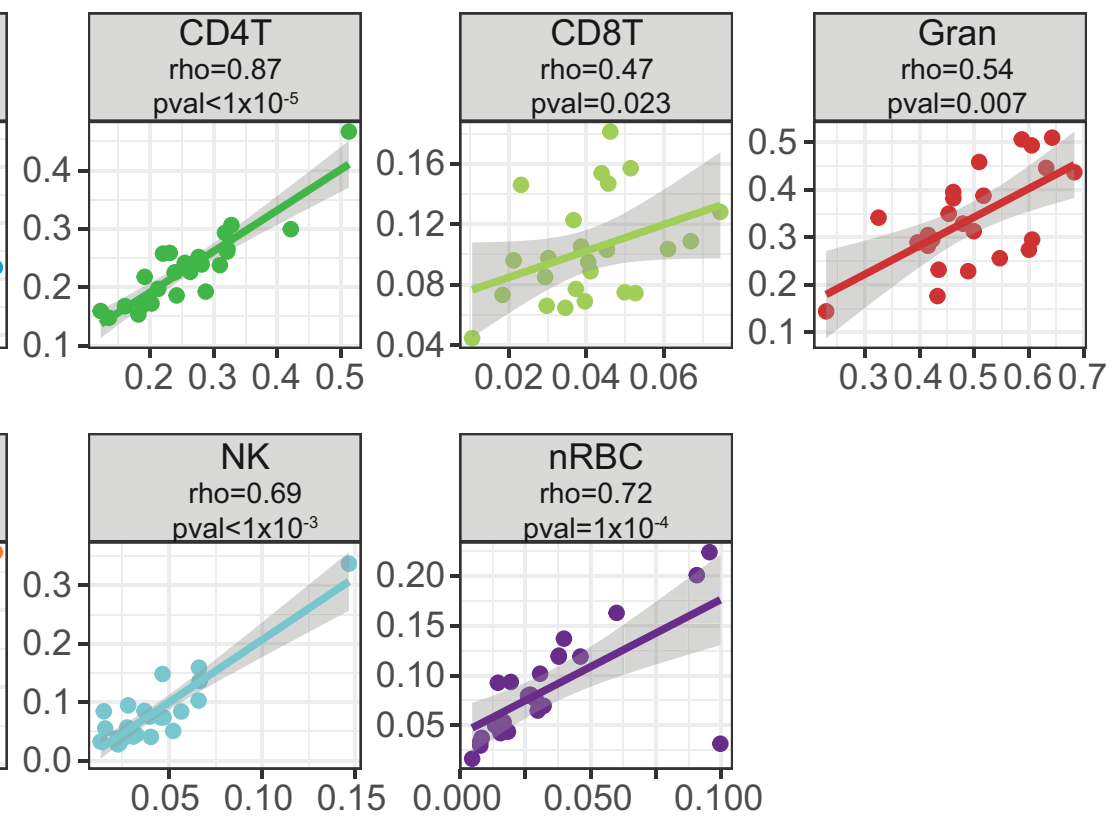

FACS measured proportion

Figure S3: Using adult references in deconvolution of cord blood results in poor prediction, and using cord references improves predictions, but some cell types remain poorly predicted. In 24 cord blood cell types, flow cytometry-based cell counts (x axis) are plotted against DNAm deconvolution-based estimates (y axis), using either adult (A) or cord (B) blood references. 
Signature Discriminability Against Other Cell Types

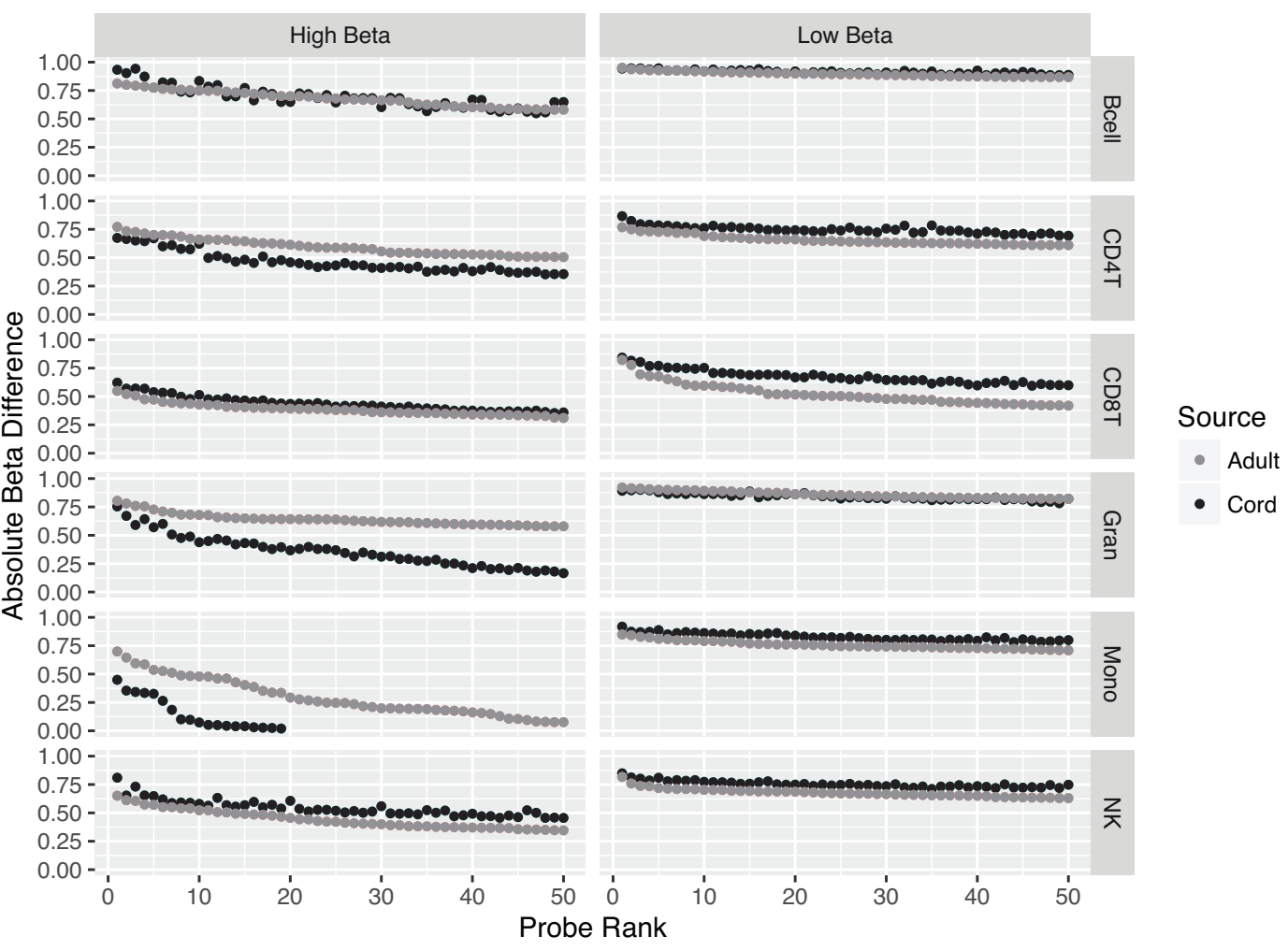

Figure S4: Cord blood had fewer cell type-distinguishing CpGs that were more methylated in a particular cell type than other cell types. Plots show the 50 best ranked distinguishing probes by $p$ value ( $x$ axis) versus the average DNAm difference between a particular cell type and other cell types (y axis), and whether they are more (top) or less (bottom) methylated in that cell type than others. For each cell type, the sites that are more methylated drop to 0 in actual DNAm difference before reaching 50, meaning that some of the probes that would have been chosen to use in deconvolution are actually not differentially methylated in that cell type at all. 

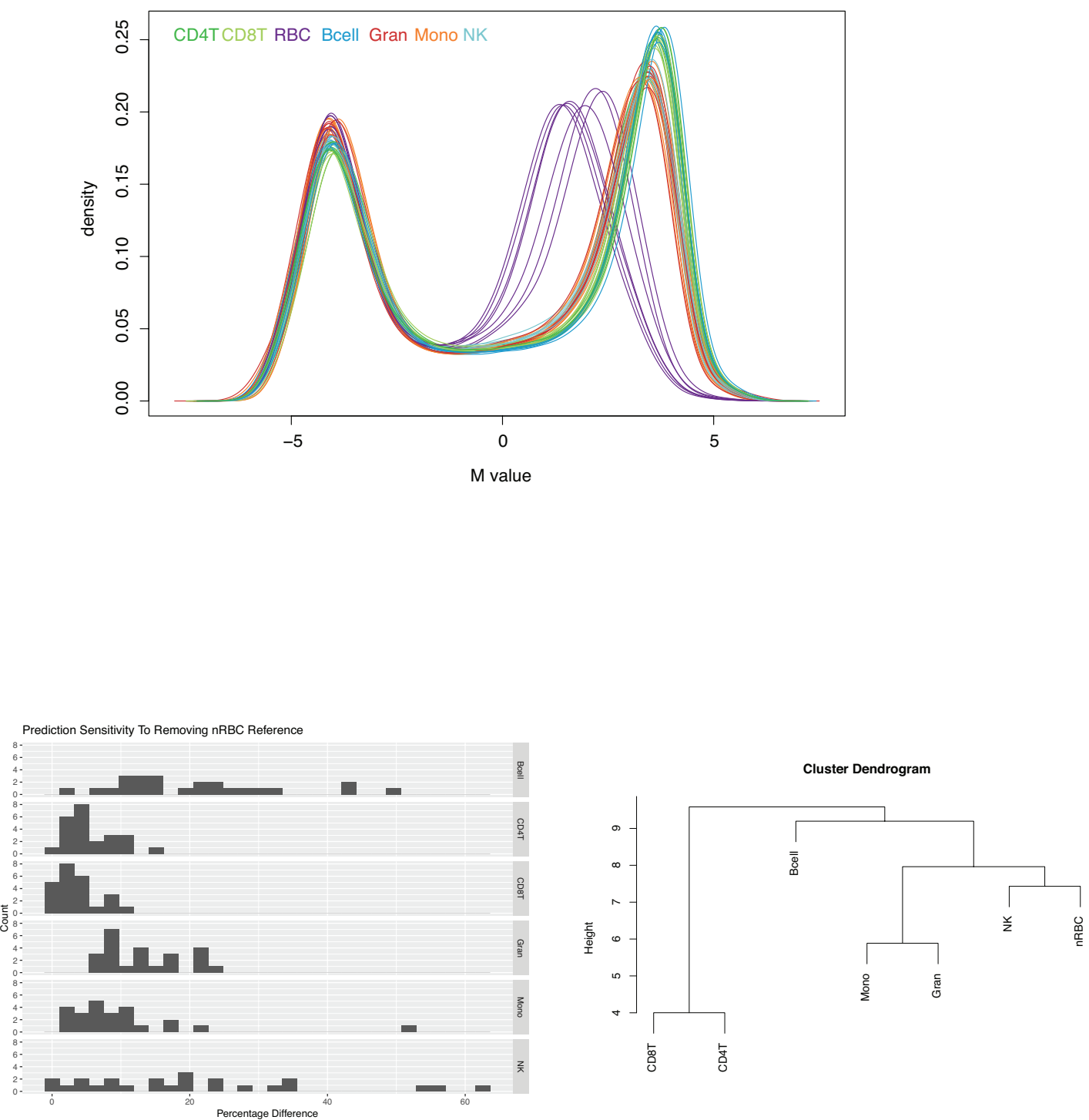
difference between predicted and actual cell counts for each cell type if $\mathrm{nRBC}$ s were not included in the prediction. C) Dendrograms showing relationships in DNAm pattern at sites used in deconvolution across the 7 cord blood cell types. NK cells are the most similar to nRBCs at these sites, explaining why this cell type is the most impacted by not including nRBCs. 
765 Table S1: Numbers of variable CpGs, defined as SD $>0.05$ in cord and adult white blood 766 cell types

\begin{tabular}{l|ll} 
Cell Type & Cord & Adult \\
\hline CD4 T & 22,755 & 34,329 \\
CD8 T & 19,848 & 85,170 \\
B & 21,100 & 78,277 \\
NK & 95,560 & 33,380 \\
Gran & 23,304 & 41,801 \\
Mono & 22,156 & 30,438 \\
nRBC & 77,888 & $\mathrm{~N} / \mathrm{A}$ \\
Mononuclear & 22,877 & 46,381 \\
cells & & \\
Whole blood & 25,910 & 42,935
\end{tabular}

768 Table S2: Number of differentially methylated sites between cord and adult and how

769 many of those are mQTLs

\begin{tabular}{|l|l|l|l|}
\hline & N unique DM sites & N mQTLs & Percentage mQTLs \\
\hline B & 6393 & 387 & 6.05 \\
\hline CD4 T & 7840 & 636 & 8.11 \\
\hline CD8 T & 4443 & 377 & 8.48 \\
\hline G & 7235 & 1355 & 18.72 \\
\hline Mo & 339 & 268 & 79.05 \\
\hline NK & 3518 & 344 & 9.78 \\
\hline Myeloid & 2062 & 65 & 11.05 \\
\hline Lymphoid & 397 & 242 & 11.73 \\
\hline
\end{tabular}

771 Table S3: Pairwise differentially methylated cord vs adult probe overlaps

\begin{tabular}{l|llllll} 
& B & CD4T & CD8T & G & Mo & NK \\
\hline B & $\mathbf{7 3 7 8}$ & & & & & \\
CD4 T & 2344 & $\mathbf{8 8 2 5}$ & & & & \\
CD8 T & 2079 & 3071 & $\mathbf{5 4 2 8}$ & & & \\
G & 2558 & 2595 & 1746 & $\mathbf{9 8 8 5}$ & & \\
Mo & 1472 & 1342 & 970 & 2650 & $\mathbf{2 9 8 9}$ & \\
NK & 2202 & 2140 & 1987 & 2081 & 1166 & $\mathbf{4 5 0 3}$
\end{tabular}

